\title{
Traumatismo lumbar por arma de fuego de proyectiles múltiples con lesión multivisceral
}

\author{
Traumatism lumbar for fire weapon of multiple \\ projectiles with multivisceral injury
}

\author{
Aimeé T Santana-Torrealba*
}

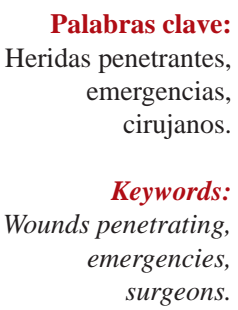

* Especialista adjunto del Servicio de Cirugía General. Hospital Universitario "Dr. Luis Razetti”. Barcelona, Estado Anzoátegui, Venezuela.

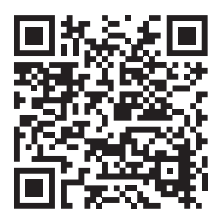

\section{RESUMEN}

Se presenta caso de trauma penetrante en región lumbar por arma de fuego de proyectiles múltiples que implicó daño en múltiples órganos, considerado una urgencia quirúrgica, aunque con presentación poco frecuente. En dichos casos el cirujano debe tomar decisiones y actuar de forma inmediata para mejorar la supervivencia del paciente. Se exponen datos clínicos, paraclínicos, su manejo y evolución, además de compararlo con escrutinio con la literatura médica relacionada.

\section{ABSTRACT}

We present a case of penetrating trauma in the lumbar region due to the multiple projectile firearm that involved damage in multiple organs, considered a surgical emergency, although with a rare presentation. In these cases, the surgeon must make decisions and act immediately to improve the survival of the patient. Clinical, paraclinical data, its management and evolution are exposed, besides comparing it with scrutiny the related medical literature.

\section{INTRODUCCIÓN}

$\mathrm{E}^{\mathrm{n}}$ n América Latina con economías y estratos sociales empobrecidos, la violencia interpersonal es la causa más frecuente de muerte e incapacidad en menores de 45 años. ${ }^{1}$ Venezuela actualmente no cuenta con registro de prevalencia de trauma, pero según el informe del año 2018 del observatorio de violencia, se registró una tasa de 81.4 muertes violentas por cada 100,000 habitantes para un total de 23,047 fallecidos en todo el territorio nacional. ${ }^{2}$

Los traumatismos lumbares penetrantes por arma de fuego de proyectiles múltiples son infrecuentes, pero se consideran una urgencia al momento de ingreso, las lesiones pueden ser complejas y afectar diversos órganos y vísceras intraabdominales, por lo que el cirujano debe actuar de forma inmediata para reducir la morbimortalidad aun en instituciones con carencias de recursos. Los estudios han demostrado que la sobrevida global se correlaciona con el número de órganos lesionados; el riesgo de morir se duplica con cada órgano lesionado adicional y la supervivencia cae significativamente cuando cuatro o más órganos están lesionados. ${ }^{3}$ Desde los últimos años del siglo XX, en los heridos graves de abdomen se aplica una estrategia de control de daños, el manejo inicial se limita a realizar una hemostasia adecuada, la eliminación de la contaminación y la prevención de la hipertensión abdominal mediante un cierre temporal. Luego, si el lesionado durante su estancia en la terapia intensiva compensa todos sus desequilibrios generales, se procede sin mayor urgencia a una o más operaciones que se planifican para la reparación definitiva de las lesiones. ${ }^{4}$ De todos los traumas penetrantes en la región lumbar, sólo 25\% tienen lesión visceral; sin embargo, es posible que haya lesiones ocultas, de modo que el mayor problema es diag-

Citar como: Santana-Torrealba AT. Traumatismo lumbar por arma de fuego de proyectiles múltiples con lesión multivisceral. Cir Gen. 2020; 42 (4): 311-315. https://dx.doi.org/10.35366/101401 
nosticar lesiones que pueden pasar inadvertidas inicialmente. Las principales lesiones por heridas penetrantes en la región lumbar son las de riñón, colon e hígado y es necesario estar atento a las manifestaciones clínicas de cada una de ellas. Se establece que los pacientes con peritonitis, estado de choque o pérdida de sangre por el tubo digestivo necesitarán laparotomía, pero las lesiones retroperitoneales, especialmente del colon, pueden pasar inadvertidas y tener manifestaciones tardías. ${ }^{5}$ En la actualidad, en los pacientes con herida penetrante en región dorsal, la primera herramienta de evaluación es el examen clínico. Si el enfermo está inestable, con peritonitis o pérdida de sangre por el recto, está indicada una laparotomía exploradora. Pero si el enfermo no cumple con los criterios de exploración quirúrgica inmediata y la evaluación clínica no es confiable, será estudiado con tomografía computarizada, según el resultado, se trasladará a unidades de observación en trauma, o unidades de cuidados intensivos, donde seguirá siendo evaluado, con vigilancia continua del estado hemodinámico del paciente con control paraclínico e imaginológico en las horas siguientes. ${ }^{6}$

\section{PRESENTACIÓN DEL CASO}

Paciente femenino de 19 años de edad con antecedentes de alergia al metamizol sódico

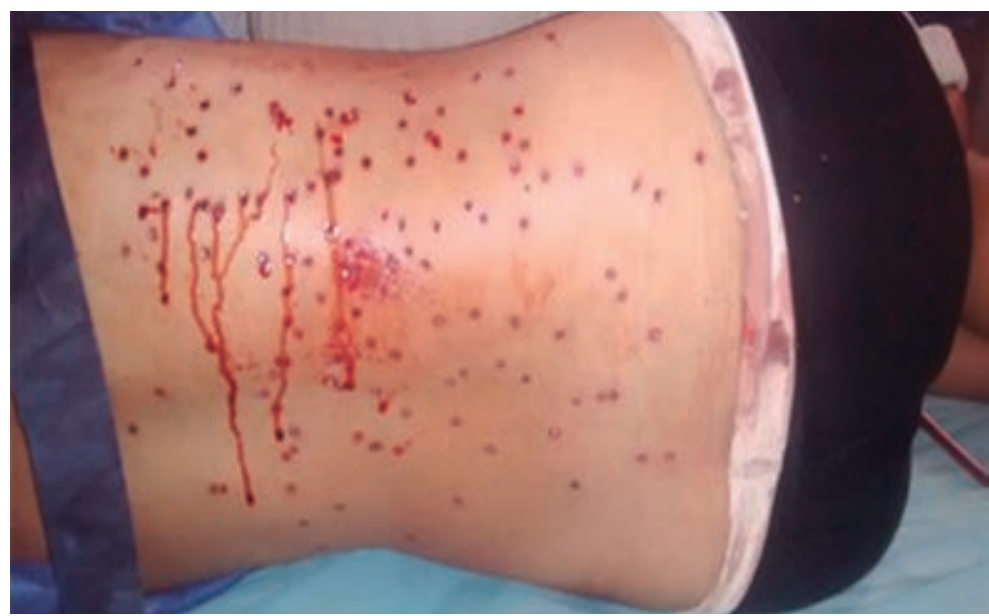

Figura 1: Paciente al momento del ingreso. Orificios de proyectil tipo descargas múltiples.

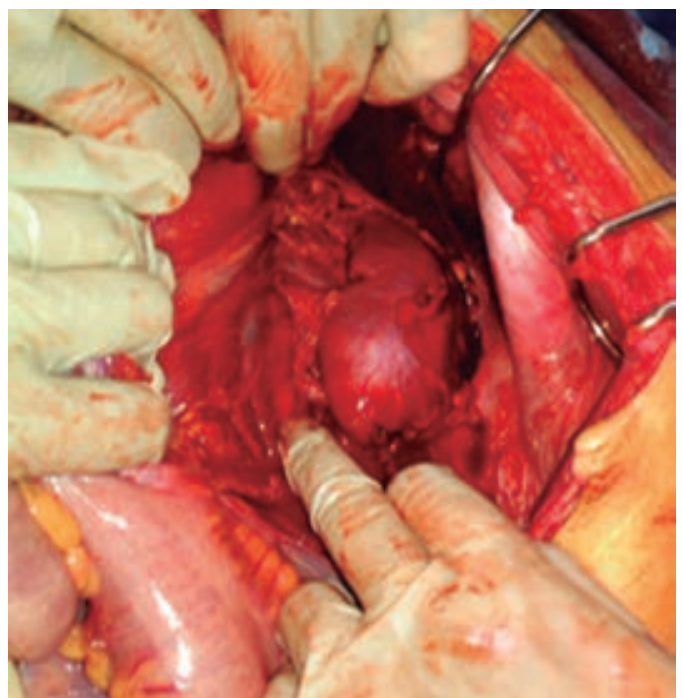

Figura 2: Lesión renal izquierda.

y cesárea segmentaria en 2015 sin complicaciones, ingresa al servicio de emergencias del Hospital Universitario "Dr. Luis Razetti" posterior a heridas múltiples por arma de fuego, en región lumbar bilateral de predominio derecho.

Examen físico: FC: 110LPM, TA: 90/50 mmHG, FR: 21RPM, T: $36.6{ }^{\circ} \mathrm{C}$.

Piel: morena, se evidencia palidez cutánea mucosa generalizada, Ilenado capilar menor de tres segundos. Cardiopulmonar: tórax simétrico, hipoexpansible a predominio de hemitórax derecho, se evidencian múltiples orificios por proyectil de arma de fuego en región inferior de hemitórax posterior izquierdo y tercio inferior de hemitórax posterolateral derecho, ruidos respiratorios disminuidos en tercio inferior hemitórax derecho sin agregados. Ruidos cardiacos rítmicos y regulares sin soplo ni galope. Abdomen: distendido, ruidos hidroaéreos disminuidos, doloroso a la palpación con signos de irritación peritoneal generalizada. Región lumbar: se evidencian orificios por proyectil de arma de fuego tipo descargas múltiples en región lumbar bilateral a predominio derecho (Figura 1). Tacto rectal: esfínter anal externo tónico, heces en ampolla, fondo de saco abombado. Extremidades: simétricas, eutróficas sin edema. Neurológico: consciente orientada en tiempo, espacio, persona. 
Es llevada a quirófano, se realiza laparotomía exploradora observando como hallazgos: 1. 1,500 $\mathrm{cm}^{3}$ de hemoperitoneo $+1,000 \mathrm{~cm}^{3}$ de sangrado transoperatorio. 2. Hematoma retroperitoneal en zona II derecha en expansión. Hematoma en zona II retroperitoneal izquierda no expansivo. 3. Múltiples lesiones $<0.5 \mathrm{~cm}$ a nivel renal derecho con compromiso del hilio. 4. Múltiples lesiones $<0.5 \mathrm{~cm}$ a nivel renal izquierdo no sangrante sin compromiso del hilio (Figura 2). 5. Dos lesiones de $0.5 \mathrm{~cm}$ de fondo vesicular. 6. Múltiples lesiones $<50 \%$ de colon ascendente hasta ángulo hepático de colon transverso. 7. Múltiples lesiones de asa delgada $<50 \%$ de $175 \mathrm{~cm}$ hasta $250 \mathrm{~cm}$ de asa fija (a $40 \mathrm{~cm}$ de válvula ileocecal). 8. Lesión $<50 \%$ de colon descendente. 9. Múltiples lesiones hepáticas puntiformes $<1 \mathrm{~cm}$ de profundidad no sangrante en segmento $\mathrm{V}$, VI, VII y VIII. 10. Lesión puntiforme de serosa en cara posterior en cuerpo de estómago. 11 . Lesión de hemidiafragma derecho de $2 \mathrm{~cm}$ hacia su inserción posterior. 12. Lesión de 1 $\mathrm{cm}$ de profundidad y $1 \mathrm{~cm}$ de longitud en polo inferior de bazo no sangrante. 13. Lesión $<0.5$ $\mathrm{cm}$ de ovario derecho no sangrante y 14 . Resto de órganos indemnes.

Por lo que se realiza: evacuación de hemoperitoneo + revisión sistemática de cavidad + maniobra de Cattell Branch Cattell-Braasch + nefrectomía derecha + maniobra de Mattox + maniobra de Kocher + colecistectomía cisticofúndica + ileotransverso anastomosis término-terminal + cierre de brecha anastomótica + rafia de colon descendente + rafia gástrica + rafia diafragmática + colocación de dren tubular pasivo de $24 \mathrm{fr}$ en fondo de saco + colocación de dren tubular pasivo de 24 fr en espacio de Morrison + colocación de dren tubular pasivo de $24 \mathrm{fr}$ en zona II retroperitoneal izquierda + colocación de puntos de tensión interna + toracotomía mínima derecha más colocación de drenaje torácico (con hallazgo de $150 \mathrm{~cm}^{3}$ de contenido hemático más aire). Recibió una unidad de concentrado globular y una unidad de sangre total durante acto operatorio, posteriormente es trasladada a la unidad de cuidados intensivos en condiciones estables. Permanece con evolución clínica satisfactoria y a las 48 horas es trasladada al área de hospitalización evidenciándose gasto de aspecto biliar por dren pasivo de nélaton dirigido al espacio de Morrison tomando muestra para citoquímico, reportando: contenido negruzco, turbio, densidad 1,015, alcalino, GR 5000, GB 1,500 NEU 64, glucosa 22, proteínas 65, Rivalta negativo, LDH 284, BT: 7.6 BD 2.5 BI 5.1, anexando diagnóstico de fístula biliar.

Es evaluada en conjunto con el servicio de nefrología, nutrición y neurocirugía, recibe durante su hospitalización tres unidades de concentrado globular, control de balance hídrico, control paraclínico, hemodinámico, de gasto por drenes y manejo de sonda pleural, más curación diaria de herida. Al quinto día de hospitalización se retira dren dirigido a fondo de saco, por ausencia de gasto. En su noveno día, en vista de salida espontánea de dren dirigido a Morrison manteniendo aún gasto biliar, se le realiza recanulación del mismo ecoguiada (Figura 3). Al décimo día se efectúa retiro de tubo de drenaje torácico por
Figura 3:

Recanulación ecoguiada de dren dirigido al Morrison.
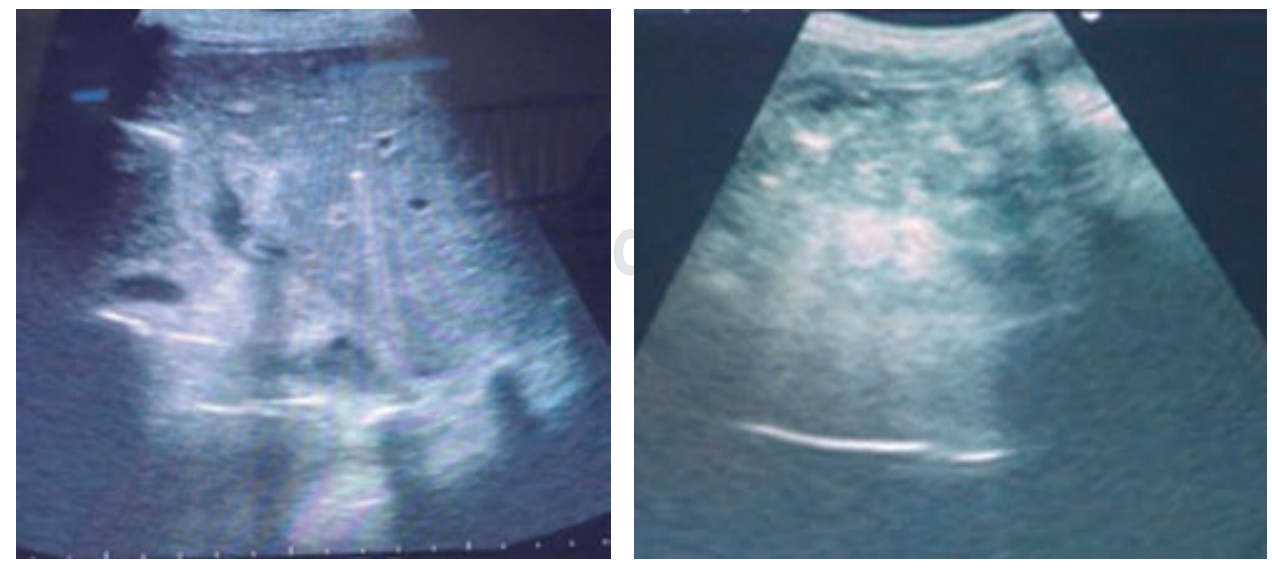

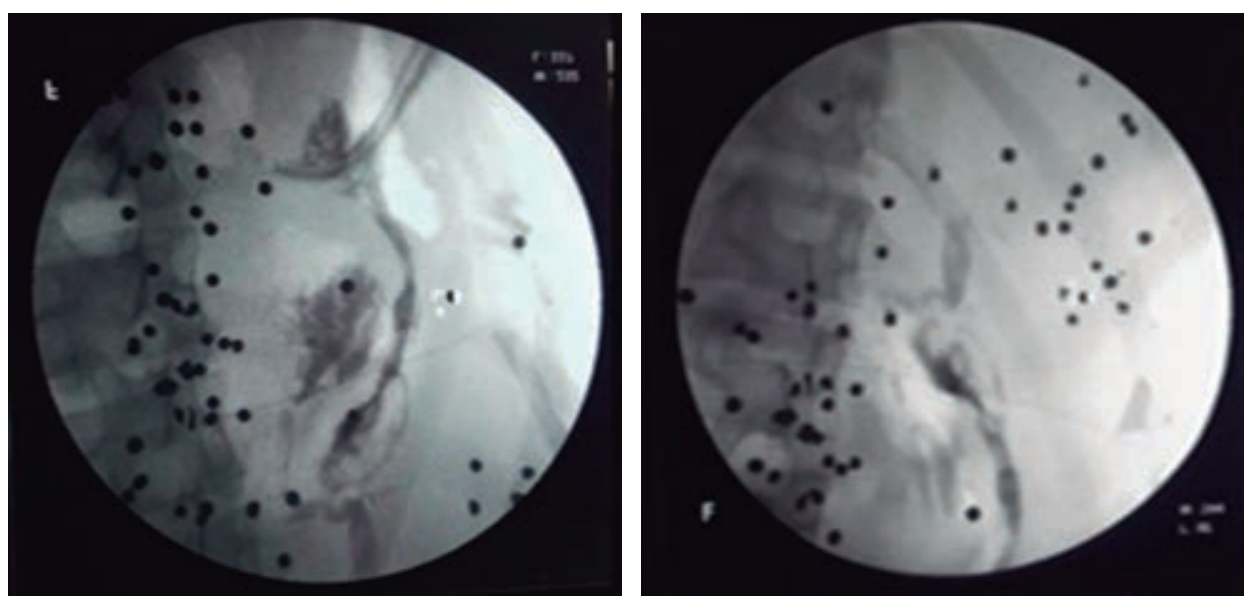

Figura 4:

Fistulografía posterior a egreso.

evidenciarse expansibilidad pulmonar. Con posterior egreso.

Control postoperatorio: se realiza fistulografía (Figura 4), se retira drenaje a los dos meses del egreso, se mantuvo con escaso gasto por cinco semanas hasta el cese del mismo.

\section{DISCUSIÓN}

En heridas por arma de fuego, Moore y Marx mencionan que $80 \%$ de las mismas son penetrantes y que en $95 \%$ de ellas hay daño visceral, ${ }^{7}$ en analogía al caso descrito, el cual posterior a traumatismo por proyectil de arma de fuego presentó lesión diafragmática, pleural, hepática, biliar, renal, gástrica, esplénica, intestinal y ovárica. Ingresa al servicio de emergencia con signos clínicos de irritación peritoneal y signos de inestabilidad hemodinámica, decidiéndose inmediatamente su intervención quirúrgica, tal y como lo recomienda un estudio realizado con 2,212 pacientes con heridas penetrantes del abdomen, tratados en tres hospitales de Charity. ${ }^{8}$ La inestabilidad hemodinámica y los signos de irritación peritoneal constituyen indicaciones absolutas para la realización de laparotomía de emergencia, los pacientes con trauma abdominal penetrante con frecuencia presentan múltiples lesiones que, además de las lesiones a órganos sólidos y estructuras vasculares, incluyen vísceras huecas, árbol biliopancreático y estructuras urológicas. ${ }^{9}$ El error estriba en demorar la intervención quirúrgica cuando es necesaria. ${ }^{10}$ Como complicación postoperatoria se evidenció y diagnosticó una fístula biliar, tratada de manera expectante con drenaje y fistulografía control. Una vez retirado dicho dren, el cierre espontáneo ocurrió a los 37 días, en concordancia con el estudio de Hollands MJ y Little JM en el que describen que el manejo expectante en pacientes con fístula biliar continúa siendo una herramienta eficiente en el paciente con traumatismo hepático severo, con una mediana de fugas biliares cerradas espontáneamente posterior a un drenaje de 33-44 días. ${ }^{11}$

\section{CONCLUSIÓN}

Todo traumatismo por arma de fuego representa una urgencia; el examen físico exhaustivo, control hemodinámico, paraclínicos y estudios de imagen como la tomografía computarizada podrán orientar y definir el tratamiento, ya sea conservador con un manejo no operatorio o una intervención quirúrgica temprana que podrá derivarse en una cirugía definitiva o en una cirugía de control de daños por etapas que disminuya la morbimortalidad del paciente. Jamás debe retrasarse el acto quirúrgico.

\section{AGRADECIMIENTOS}

Agradezco al Servicio de Cirugía General del Hospital Universitario "Dr. Luis Razetti" por disponer de sus espacios para el desarrollo del proceso de este estudio. Al personal del postgrado de Cirugía General en especial a la Dra. Katherine Rodríguez por su valiosa participación y contribución. 


\section{REFERENCIAS}

1. Andreani HD, Crosbie G, Fontanet N, Hernan R y Vozzi J. Traumatismos penetrantes toracoabdominales por heridas de arma de fuego. Utilidad de la videolaparoscopia. Rev Argent Cirug. 2017; 109: 17-20.

2. OVV-LACSO. Informe anual de Violencia 2018. Observatorio Venezolano de violencia. Venezuela. [Fecha de consulta: 02 de Enero de 2019]. Disponible en línea: https://observatoriodeviolencia.org.ve/ovvlacso-informe-anual-de-violencia-2018/

3. Nicholas JM, Rix EP, Easley KA, Feliciano D, Cava $\mathrm{R}$, Ingram $\mathrm{WL}$, et al. Changing patterns in the management of penetrating abdominal trauma: the more things change, the more they stay the same. J Trauma. 2003; 55: 1095-1110.

4. Camilo A, Hernández M, Hidalgo M, Borges R y Montejo J. Manejo emergente de heridas lumbares. Medicina de Urgencias, Cirugía General y Digestiva, Artículos. Cuba 2011. [Fecha de consulta: 10 de Julio de 2018]. Disponible en línea: https://www. portalesmedicos.com/publicaciones/articles/3360/1/ Manejo-emergente-de-heridas-lumbares.html
5. Espinoza R. Manejo de las heridas penetrantes lumbares: operar o no operar. Medwave. 2006; 6: e1243.

6. Cobo P, Caballero R, Hernando Á. Lesión penetrante de espalda y flancos. Emergencias. 1995; 7: 223-230.

7. Moore EE, Marx JA. Penetrating abdominal wounds. Rational for exploratory laparotomy. JAMA. 1985; 253: 2705-2708.

8. Cohn I, Ingram JC, Johnson LW, Nance FC, Wenner $\mathrm{MH}$. Surgical judgment in the management of penetrating wounds of the abdomen: experience with 2,212 patients. Ann Surg. 1974; 176: 639-646.

9. Pinedo-Onofre AJ, Guevara-Torres L, Sánchez-Aguilar MJ. Trauma abdominal penetrante. Cir Ciruj. 2006; 74: 431-442.

10. Peterson NE. Genitourinary trauma. In: Mattox KL, Feliciano DV, Moore EE (eds). Trauma. 4th ed., Chapter 37, New York: McGraw-Hill, 2000, 839-879.

11. Hollands MJ, Little JM. Post-traumatic bile fistulae. J Trauma. 1991; 31: 117-112.

\section{Correspondencia:}

Aimeé T Santana-Torrealba

Barcelona, Avenida Rotaria,

Barcelona 6001, Anzoátegui.

E-mail: aimeesantana01@gmail.com 